\title{
A Recommendation Method of National Fitness Items Based on Neural Network Algorithm
}

\author{
Liangming $\mathrm{Li}^{1}$ and Aihua Yang $\mathbb{D}^{2}$ \\ ${ }^{1}$ School of Physical Education, Hunan University of Science and Technology, Xiangtan, 411201, China \\ ${ }^{2}$ School of Physical Education, Changsha University of Science and Technology, Changsha, 410076, China \\ Correspondence should be addressed to Aihua Yang; 001962@csust.edu.cn
}

Received 12 November 2021; Revised 28 November 2021; Accepted 3 December 2021; Published 18 January 2022

Academic Editor: Le Sun

Copyright (c) 2022 Liangming Li and Aihua Yang. This is an open access article distributed under the Creative Commons Attribution License, which permits unrestricted use, distribution, and reproduction in any medium, provided the original work is properly cited.

\begin{abstract}
In order to improve the effectiveness of national fitness programs, this article analyzes the method of national fitness items recommendation based on a neural network algorithm. By using the time and space characteristics of fitness users' sign-in, a novel POI recommendation model is proposed, and a novel fusion method is proposed to combine similarity and spatial similarity to achieve the final similarity calculation based on fitness users' temporal and spatial preferences. In addition, in order to model the spatial similarity of fitness users, the Voronoi diagram is constructed by using the geographic locations of all POIs. Finally, this paper constructs a recommendation system for national fitness items based on a neural network algorithm. The experimental research results show that the national fitness program recommendation system proposed in this article basically meets the expected demand.
\end{abstract}

\section{Introduction}

The implementation of the national fitness program and the development of mass sports have improved the overall quantity and quality of China's sports population. However, only participating in physical exercises cannot meet people's fitness needs. The development of sports events, especially mass sports events, should be enriched. The relationship between mass physical exercises and mass sports events complements and promotes each other. Mass sports events are the display of the results of mass sports exercises, the best test of fitness activities, can better motivate and drive the masses to participate in sports exercises, and play a "leveraging" role in setting off a mass sports climax. Mass sports are the basis for conducting mass sports events, and they support and guarantee the development of mass sports events [1]. Mass sports events set off a fitness craze among the masses, allowing them to show their strong physiques and fighting spirit, while also forming a carnival that focuses on participation, national fitness, and happy sports. Moreover, they promote the improvement of the masses' awareness of physical exercise and the development of lifelong physical exercise habits, so that fitness can be integrated into the daily life of the masses [2].

National fitness is an important foundation for building a healthy China and an inevitable requirement for the people to yearn for a better life. The improvement of the national fitness system can enhance the country's overall national strength and reflect the level of social civilization. This study conducted research on the construction of the nationwide fitness system, deeply explores the relevant laws, regulations, policy documents, etc. promulgated by China for the development of nationwide fitness programs and finds the problems and shortcomings in the development of nationwide fitness programs. This has important practical significance for improving the construction of the nationwide fitness system, fully implementing the nationwide fitness strategy, and accelerating the construction of a sports power [3].

With the continuous development and progress of the times, the domestic economic development continues to accelerate, people's income is further increased, the quality 
of life is improved, and people's needs for quality of life and sports activities are increasing. However, from the current basic situation, there are still many problems in public sports services, and the quality level cannot meet actual needs. With the holding of the Olympic Games, the whole people's love for sports has set off a frenzy on the land of China. The manifestation of this contradiction is very prominent.

With the continuous development and progress of the times, people have more and more demands for quality of life and sports activities. However, judging from the current domestic basic situation, there are serious deficiencies in the supply and construction of related infrastructure. Everyone has a relatively limited number of fitness venues. The common problem is that the existing fitness venues are not used efficiently at this stage. The popularity of fitness education among people is generally low. There are no unified system standard, legal conditions, and scientific and reasonable fitness requirements for the development of fitness among the whole people. It is necessary to continuously rationalize the operation mode and operation mechanism of the national fitness program and improve and innovatively deepen reforms.

According to the actual needs of the national fitness program, this paper combines the neural network algorithm to construct the national fitness program recommendation system to improve the recommendation efficiency of the national fitness program.

\section{Related Works}

For the understanding of the national fitness program's mechanism, Ferguson et al.'s [4] point of view is that it can provide all the people with basic conditions for physical exercise activities and the creation of the environment to meet public's basic requirements for physical fitness. The physical quality has been significantly improved, and the related services and guarantee systems are produced by it. Kimasi et al.'s [5] view is that the national fitness system should be a system that has a direct impact on and restricts the participation of all people in fitness activities, comprehensively improves the overall physique of the people, and serves, adapts, and guarantees a comprehensive physique. The basic structural framework of the mechanism is the organization, activity, and material guarantee related to fitness activities. There are many support systems that can meet diversified and different requirements at the same time. Service and management are as important as the integrity structure, openness, and effectiveness. Literature [6] conducts research and analysis on the relevant systems of social sports instructors. The system mechanism of sports instructors has two parts: "Social Sports Instructor Technical Grade System" and "Social Sports Instructor National Professional Standards." The research direction of the literature [7] is to focus on the basic status of fitness for all the people in China. Through investigation and analysis, it is concluded that the problems in the process of fitness for the whole people are as follows: the source of funds is relatively single, there is no professional social nature, sports instructors, there is no conscious structure of participating in physical exercises in the subconsciousness of farmers [8], and the sports management mechanism in rural areas is very backward [9]. The research direction of the literature [10] is the characteristics of the population participating in sports. In terms of gender, men are generally higher than women. In terms of geographic composition, cities are generally higher than rural areas and the east is higher than the west. In terms of age, the two ends are taller, and the middle is shorter like a pommel horse-shaped structure. At the same time, it also clearly pointed out that the entry point for the improvement of the demographic structure in sports is the country's policy solution to social problems.

When the researcher Dong Li analyzed, according to the relevance of content and level, the formation of the national fitness system was divided into categories [11]. Divided according to the level, the national fitness mechanism can be divided into national and provincial levels in different regions, and a more detailed national fitness system should be built in the city to form the entire national fitness-related mechanism in the city [12]. To distinguish from the level, the content structure of the national fitness system is composed of basic services and basic guarantees, and a comprehensive analysis on the existing relationship level should be conducted. Comprehensive research on various elements should be conducted, and a reasonable combination should be carried out. Continuous improvement of the operation form and operation system of the fitness business and coordination of all the elements should be carried out, so as to give full play to the basic role of the national fitness operation mechanism [13]. According to the analysis carried out by Mountjoy et al. [14], the factors that influence the national fitness service mechanism include different types. The basic content can be summarized as the formulation and implementation of policies and systems, the arrangement and organization of fitness activities, the investment of funds, and so on.. Regarding the supply and construction of related infrastructure, the collection and analysis of information and materials, etc., through the research and analysis of these levels, we can more scientifically and reasonably grasp the operating mechanism related to national fitness. Pulido et al. [15] points out that the establishment of a national fitness service mechanism needs to focus on research and development from the following five aspects: publicity, construction of facilities and equipment, scientific guidance, dynamic monitoring, and organizational management.

\section{A Recommendation Method of National Fitness Items Based on a Neural Network Algorithm}

This paper proposes a novel POI recommendation model by using the time and space characteristics of fitness users' sign-in. The POI recommendation problem is defined as follows: when the sign-in record of the target fitness user is 
given, the main task of POI recommendation is to recommend the top-k positions that may be of interest to the fitness user. Table 1 lists some symbols that need to be used.

The model implements the POI personalized recommendation problem. The general idea is as follows: after the target fitness user $u_{i}$ is given, the system first searches for a collection of fitness users with similar temporal and spatial characteristics to the target fitness user $u_{i}$. In addition, the personalized recommendation system should not set a fixed $\mathrm{K}$-nearest neighbor value for all fitness users. Therefore, our system has proposed a set of adaptive K-nearest neighbor algorithm, which can select different K-nearest neighbors for different fitness users, so as to provide personalized location recommendation services for fitness users. Finally, based on the idea of collaborative filtering, suitable points of interest are selected and recommended to target fitness users.

Our model is designed with two modules: a fitness user similarity module that integrates temporal and spatial characteristics and a point-of-interest recommendation module. The details of these two modules will be introduced below.

In order to find fitness user groups with temporal and spatial similarity to the target fitness user, this research will model the temporal and spatial characteristics of fitness users, respectively, and then, design a personalized calculation method of fitness user similarity.

Generally, fitness users display different preferences at different time intervals. Each time interval is represented by a time ID, and the timestamp of each check-in record belongs to an interval. In order to model the time characteristics of fitness users, we construct a third-order tensor $X \in R^{U \times T \times C}$, where $U, T$, and $C$ represent the number of fitness users, the number of time intervals, and the number of categories, respectively.

The tensor decomposition can be used to alleviate missing or sparse data and explore the potential associations of fitness users, time, and POI categories. To avoid those negative values which have no meaning for the preference measure during the restoration process, we add non-negative constraints in the decomposition process. The decomposition formula is as follows:

$$
X=G \times{ }_{1} A \times{ }_{2} B \times{ }_{3} C .
$$

After non-negative tensor decomposition, we obtain a time-related preference matrix for each fitness user, as shown in Figure 1. In order to more accurately infer the time preference characteristics of fitness users, we normalize the sum of each row of the matrix. Therefore, the matrix shows a certain fitness user's preference for POI categories at different time intervals.

We use $P T_{i}$ to represent the time category preference matrix of the fitness user $u_{i} . P T_{i}(1,1)$ represents the access probability of the fitness user $u_{i}$ accessing the POI of the category $c_{1}$ at a time interval $t_{1}$. Therefore, the similarity of preferences of different fitness users based on time is expressed as follows [16]:
TABLE 1: Symbol description.

\begin{tabular}{lc}
\hline Symbol & Illustrate \\
\hline$U$ & Collection of all fitness users \\
$u_{i}$ & The i-th fitness user in $U$ \\
$P$ & Collection of all POIs \\
$p_{i}$ & The i-th sign-in point in $P$ \\
$S_{i}$ & Collection of check-in points visited by the fitness user $u_{\mathrm{i}}$ \\
$P T$ & Time category preference matrix of fitness users \\
$K$ & Number of similar fitness users \\
\hline
\end{tabular}

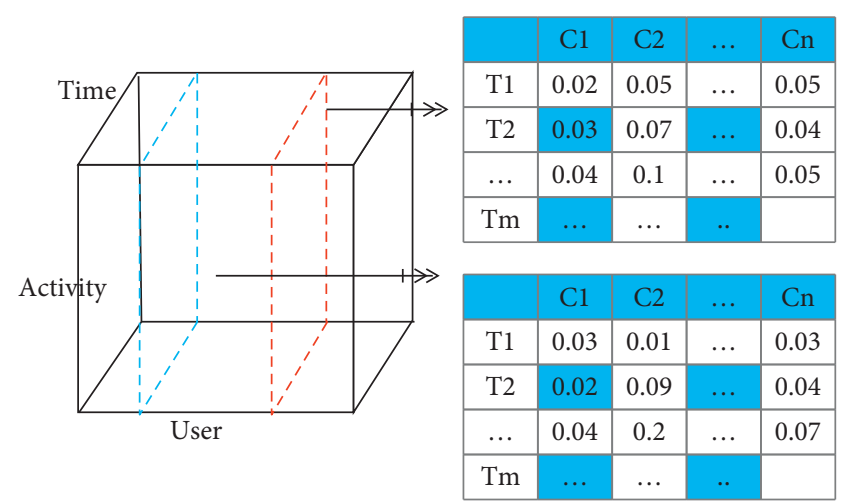

Figure 1: Time influence modeling.

$$
\varphi_{u_{i}, u_{j}}=\frac{\sum\left(P T_{i} \odot P T_{j}\right)}{\sqrt{\sum_{s_{i, k} \in P T_{i}} s_{i, k}^{2}} \sqrt{\sum_{s_{j, k} \in P T_{j}} s_{j, k}^{2}}} .
$$

In order to model the spatial activity preferences of fitness users, we use the geographic locations of all POIs to construct a Voronoi diagram. Specifically, as shown in Figure 2, the entire city is divided into multiple Voronoi grids. Therefore, a fitness user's spatial activity preference can be reflected by including a set of POIs that he has visited.

We use the Voronoi diagram to check the geographic check-in locations of fitness users because the Voronoi diagram can define whether two POIs are adjacent or not more reasonably than the Euclidean distance. In different areas of the city, the distribution of interest points of fitness users when signing in may be sparse or dense. Obviously, in sparse areas, the area of Voronoi grid cells is relatively large, while in dense areas, where check-in points are distributed, the area of Voronoi grid cells is relatively small. Therefore, it can provide an adaptive estimate of the distance between POIs.

After the Voronoi diagram is constructed, we can measure the similarity of the spatial activities of the two fitness users by exploring whether there are overlaps or adjacent Voronoi grid units in the check-in records of the two fitness users. For two fitness users $u_{i}$ and $u_{j}$, our steps to calculate the spatial similarity are as follows:

(1) For each check-in point $p_{r}$ in the fitness user checkin set, we use formula (3) to calculate the similarity of each check-in point $p$ [17]: 


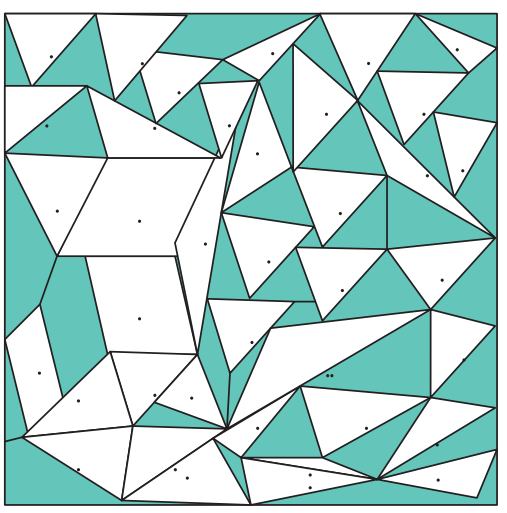

Figure 2: Example of a Voronoi diagram.

$$
y_{r}=\log \left(\frac{|U|}{n_{r}}\right)
$$

Among them, $U$ represents the total number of fitness users in the check-in set, and $n_{r}$ is the number of fitness users who have visited $p_{r}$.

(2) The algorithm constructs an array $Y$ to store the similarity of each POI and uses the minimum and maximum normalization method to realize the normalization of $\mathrm{Y}$.

(3) The algorithm constructs set $S_{i}$ and $S_{j}$ to store fitness users $u_{i}$ - and $u_{j}$-visited check-in points, respectively. The algorithm constructs set $A$ and $B$ such that $A=$ $S_{i} \cap S_{j}$ and $B=S_{i} \cup S_{j}$.

(4) The algorithm constructs a set $C$ such that $C=B-A$. The $D$ set is a subset of the $C$ set. The $D$ set stores each pair of adjacent POIs visited by fitness users $u_{i}$ and $u_{j}$ in the Voronoi diagram. Therefore, we use formula (4) to count the spatial similarity between $u_{i}$ and $u_{j}$ [18]:

$$
\omega_{u_{i}, u_{j}}=\frac{\sum_{p_{r} \in A} y_{r}+1 / 2 \sum_{\left\{p_{m}, p_{n}\right\} \in D}\left(y_{m}+y_{n} / 2\right)}{|B|} .
$$

After two fitness users $u_{i}$ and $u_{j}$ are given, we first calculate the time similarity and spatial similarity, respectively. A novel fusion method is proposed to combine them together to realize the final similarity calculation based on the temporal and spatial preferences of fitness users. Our model uses two weights to reflect the influence of time preference and spatial preference characteristics, respectively. The specific calculation is as

$$
\operatorname{sim}_{\left(u_{i}, u_{j}\right)}=\alpha \varphi_{u_{i}, u_{j}}+\beta \omega_{u_{i}, u_{j}}
$$

By using our fusion similarity calculation method, the similarity between each pair of fitness users can be obtained. We set the number of neighbors $K=\{1,2,3,4, \ldots, 20\}$ and set the fitness user $u_{i}$. According to the top $\mathrm{K}$ most similar fitness users, the recommended score for each POI can be calculated as follows:

$$
\begin{aligned}
\operatorname{score}(i, j, k) & =\sum_{u_{q} \in K} \operatorname{sim}\left(u_{i}, u_{q}\right) \times r_{q, j}, \\
r_{q, j} & =\operatorname{Min}_{i}+\frac{\operatorname{Max}_{i}-\operatorname{Min}_{i}}{\operatorname{Max}_{q}-\operatorname{Min}_{q}} \times\left(f_{q, j}-\operatorname{Min}_{q}\right) .
\end{aligned}
$$

In formula (7), $f_{q, j}$ represents the access probability of the fitness user $u_{q}$ at the check-in point $p_{j}$. In formula (6), $r_{q, j}$ is the result of the normalization of $f_{q, j} . r_{q, j}$ represents the pseudoscoring of the fitness user $u_{q}$ on the $p_{j}$ at the check-in point. The range of the check-in probability of the fitness user $n$ at each POI is between $\operatorname{Max}_{i}$ and $\operatorname{Min}_{i}$.

In order to improve the accuracy of the recommendation, we use an adaptive K-nearest neighbor algorithm to determine the value of $\mathrm{K}$. That is, for different fitness users, we select a different number of similar fitness users to generate the corresponding recommendation list. The specific steps of our algorithm are as follows:

(1) For different $\mathrm{K}$ value selections, we use formula (8) to calculate the corresponding recommendation error:

$$
\text { error }_{i}=\sum_{j \in S_{i}} \frac{\left|r_{i, j}-\operatorname{score}(i, j, k)\right|}{\left|S_{i}\right|} .
$$

In formula (8), $r_{i, j}$ represents the pseudorating of the fitness user $u_{i}$ on the check-in point $p_{j}$. Score $(i, j, k)$ represents the recommendation score of the fitness user $u_{i}$ on the check-in point $p_{j}$ based on the top $\mathrm{K}$ most similar fitness users. $S_{i}$ represents the records of POIs actually visited by fitness users in the training set.

(2) The algorithm selects the K value that causes the least error as the number of the most similar fitness user groups to recommend points of interest.

Then, we propose the next POI recommendation system. We will describe the overall framework of the model. As shown in Figure 3, our next POI recommendation system consists of two main parts: a context-aware similarity calculation module and a point-of-interest recommendation module.

For each fitness user, we connect all check-in records to construct a virtual trajectory of the fitness user. Therefore, each fitness user has six virtual trajectories corresponding to 6 time intervals.

Figure 4 shows the construction of a virtual trajectory for fitness users. The Voronoi diagram is constructed by using three kinds of information: time, latitude, and longitude. Moreover, the constructed trajectory is not the real exercise trajectory of the fitness user but a virtual trajectory constructed by Voronoi, and the virtual trajectory is only used for the calculation of the similarity of the fitness user.

We set a target fitness user $u_{i}$. $t_{k}$ is the geographic location of the check-in point $p_{k}$, and $c_{k}$ represents a specific category of the check-in point $p_{k}$ [19].

We first set a virtual trajectory for the target fitness user $u_{i}$. 


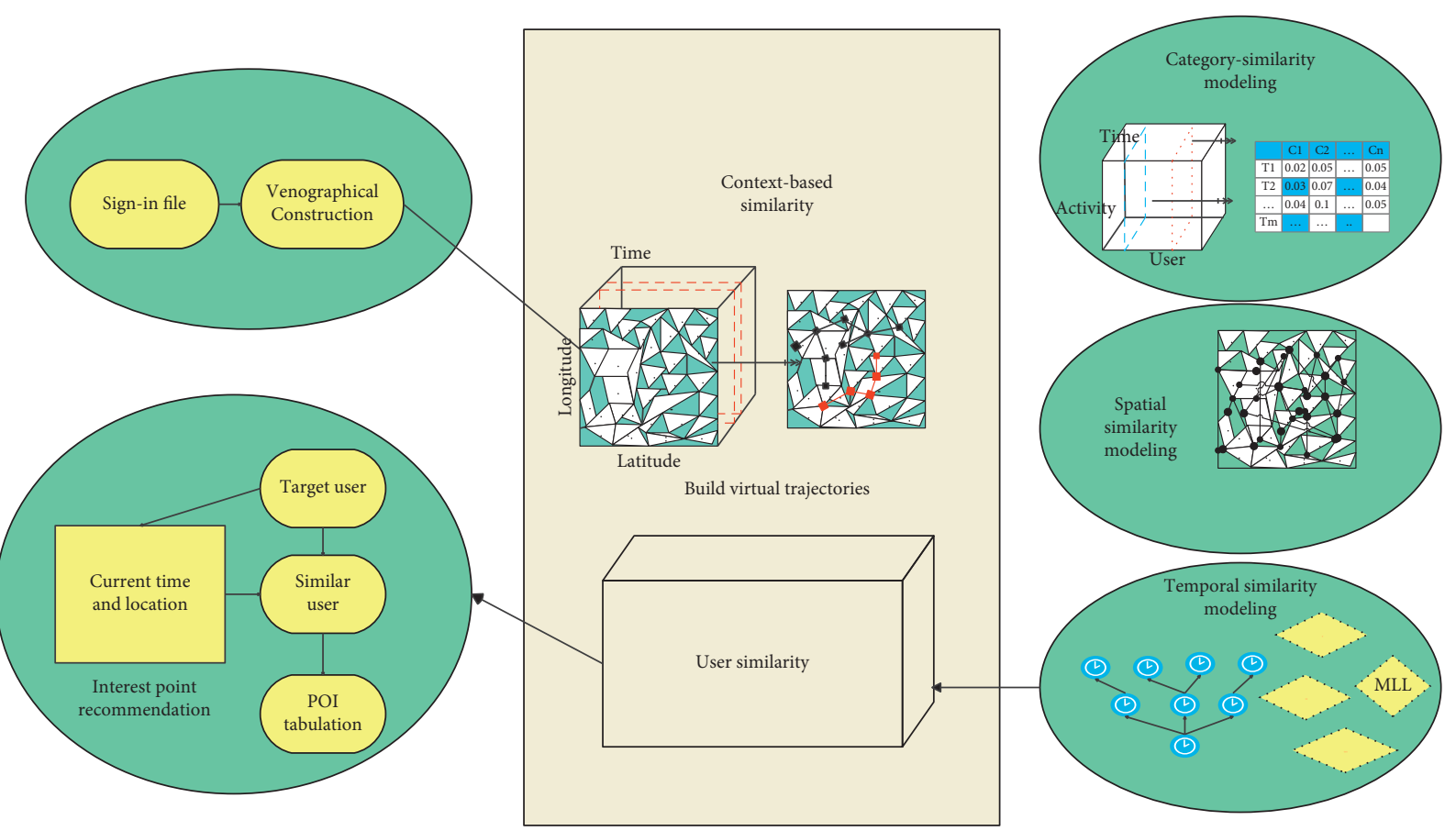

FIGURE 3: Schematic diagram of the system model.

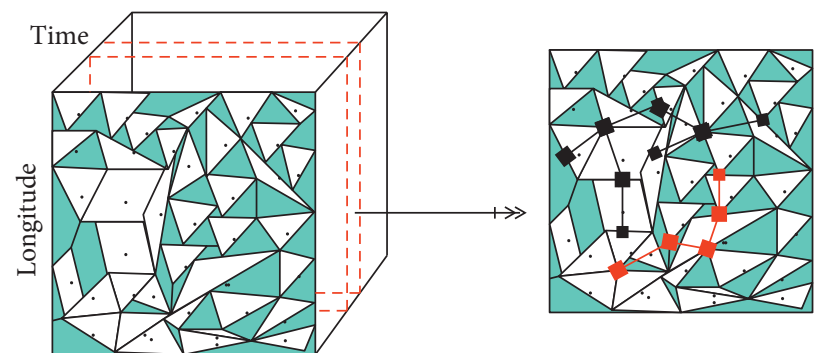

Latitude

FIgURE 4: Example of construction of the virtual trajectory for fitness users.

We divide the virtual trajectory of the fitness user $u_{i}$ into $n$ groups. The detailed steps are as follows:

(1) The algorithm constructs an empty set group for $_{k}$ each sign-in point $p_{k}\left(p_{k} \in L_{q}^{i}\right)$ of the fitness user $u_{i}$.

(2) The algorithm constructs a virtual trajectory for the fitness user $u_{j}$, denoted by $L_{q}^{j}$.

For each check-in point $p_{k}\left(p_{k} \in L_{q}^{i}\right)$, the algorithm calculates $\left|t_{m}-t_{k}\right|$.

The algorithm compares each time distance and finds the check-in point $p_{m n}$ closest to $p_{m}$ in the check-in time, where $p_{\text {min }} \in L_{q}^{i}$.

Figure 5 shows an example diagram of the trajectory division, and there may be empty sets in these groups.

By considering the time characteristics, our method successfully converts the trajectory similarity calculation into the similarity calculation between each check-in point $p_{x}$ and the check-in point in the corresponding group. The specific similarity calculation formula is as follows:

$$
\begin{aligned}
\operatorname{simuser}\left(u_{i}, u_{j}\right) & =\frac{\sum_{k=1}^{n} \operatorname{sim}\left(p_{k}, \operatorname{grou} p_{k}\right)}{n}, \\
\operatorname{sim}\left(p_{k}, \operatorname{grou}_{k}\right) & =\frac{\sum_{p_{m} \in \text { group }} \operatorname{simpoint}\left(p_{k}, p_{m}\right)}{\left|\operatorname{grou} p_{k}\right|} .
\end{aligned}
$$

Specifically, in formula (9), $\operatorname{simuser}\left(p_{k}, \operatorname{sroup}_{k}\right)$ represents the fitness track between fitness users $U_{i}$ and $U_{j}$ in a given time interval. $\mathrm{n}$ represents the virtual fitness track length of the fitness user $u_{i}$. $\operatorname{sim}\left(p_{k}, \operatorname{sroup}_{k}\right)$ represents the similarity between the fitness location $p_{k}\left(p_{k} \in L_{q}^{i}\right)$ and its corresponding set. In formula $(10), \operatorname{sim}\left(p_{k}, \operatorname{sroup}_{k}\right)$ represents the similarity between the fitness location $p_{k}\left(p_{k} \in L_{q}^{i}\right)$ as well as $p_{m}\left(p_{k} \in \operatorname{group}_{k}\right)$ of the fitness user $\mathrm{u}[20]$.

To model the spatial similarity of fitness users, we construct the Voronoi diagram. Each unit contains one and only one POI. Therefore, the spatial-related preferences of fitness users can be reflected by including the POIs they have visited. The Voronoi diagram defines whether there is a spatial proximity relationship between two POIs more 


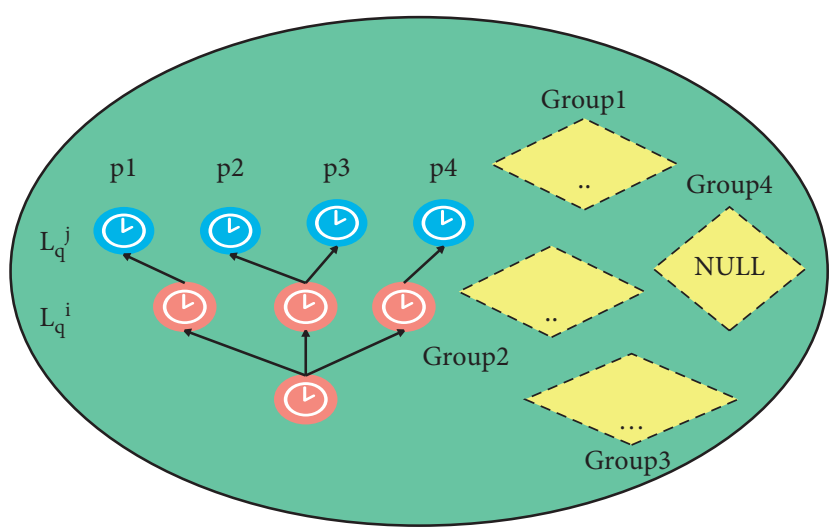

FIGURE 5: Schematic diagram of fitness user trajectory grouping.

reasonably than the Euclidean distance, and it can provide an adaptive estimate of the distance between POIs.

The calculation method of the geographic similarity based on the Voronoi diagram is described. When the time interval $\mathrm{T}_{\mathrm{x}}$ is given:

(1) The algorithm determines the set of adjacent points. They are defined as adjacent points.

(2) The algorithm adds an undirected edge.

We use equation (11) to calculate the spatial similarity:

$$
\omega\left(p_{k}, p_{m}\right)= \begin{cases}1, & s=0, \\ \frac{2^{-d}}{2^{s-1}}, & s \geq 1 .\end{cases}
$$

Figure 6 shows a constructed undirected graph. In Figure 6, the shortest path length from $p_{k}$ to $p_{m}$ is 4 , so $s=4$.

First, the algorithm constructs a fitness user category sign-in matrix and then uses the matrix factorization technology to recover the lost data.

After obtaining a fitness user category sign-in matrix, $C$ represents the total number of categories. Therefore, each column of $Q$ represents the check-in frequency of fitness users in a certain category, which can be regarded as the feature vector of the category.

For each sign-in point $p_{k}\left(p_{k} \in L_{q}^{i}\right)$,

$$
\psi\left(p_{k}, p_{m}\right)=\frac{\overrightarrow{c_{p_{k}}} \cdot \overrightarrow{c_{p_{m}}}}{\sqrt{\left|\overrightarrow{c_{p_{k}}}\right|^{2}} \times \sqrt{\left|\overrightarrow{c_{p_{m}}}\right|^{2}}} .
$$

In formula $(12), \overrightarrow{c_{p_{k}}}$ and $\overrightarrow{c_{p_{m}}}$ represent the category feature vectors corresponding to their check-in points, respectively.

After a given target fitness user $u_{i}$, we calculate contextual perception similarity between the fitness user $u_{i}$ and other fitness user $u_{j}$. The detailed steps are as follows:

(1) The algorithm constructs group $\mathrm{k}$ as shown in the previous section. Each sign-in point $p_{m}\left(p_{m} \in \operatorname{group}_{k}\right)$ and $p_{k}$ share similar time characteristics. Repeat the following operations until all check-in points in group $_{\mathrm{k}}$ are traversed:

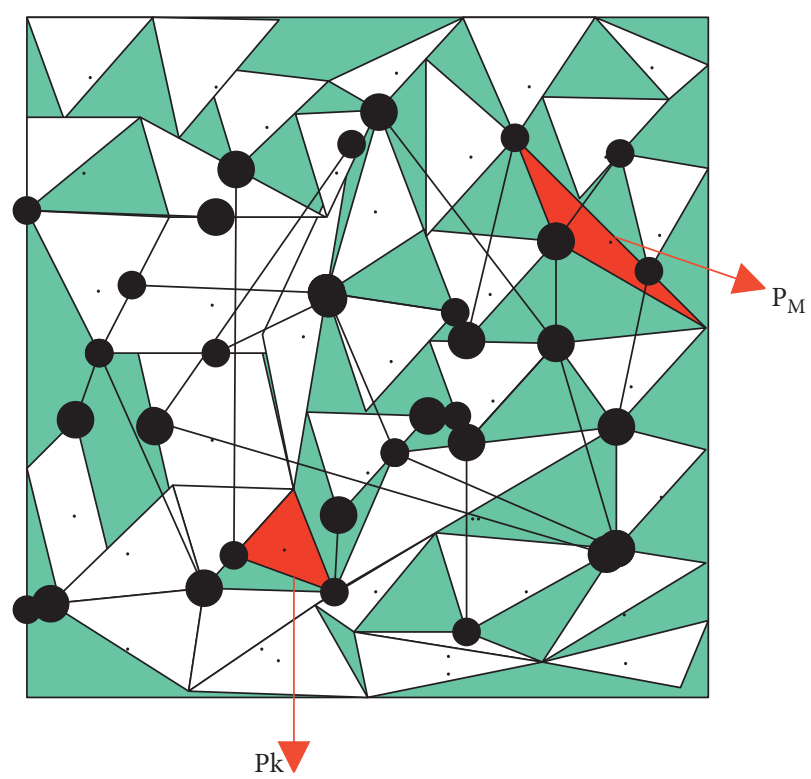

FIGURE 6: Spatial distance modeling for fitness users.

(i) The algorithm uses formula (11) to calculate the spatial similarity $\omega_{\left(p_{k}, p_{m}\right)}$ between sign-in points $p_{k}$ and $p_{m}$ and uses formula (12) to calculate the category similarity $\psi_{\left(p_{k}, p_{m}\right)}$ between sign-in points $p_{k}$ and $p_{m}$.

(ii) The algorithm uses formula (13) to calculate the overall similarity between sign-in points $p_{k}$ and $p_{m}$.

$$
\operatorname{simpoint}\left(p_{k}, p_{m}\right)=\psi\left(p_{k}, p_{m}\right) \psi_{\left(p_{k}, p_{m}\right)} \text {. }
$$

(2) The algorithm calculates the similarity between the sign-in point $p_{k}$ and its corresponding set group $p_{k}$ by using formula (11).

The algorithm calculates the context-aware similarity between the fitness user $u_{i}$ and fitness user $u_{j}$ by using formula (9).

After calculating the similarity between the target fitness user $U_{i}$ and other fitness user $U_{j}$, we construct a Sui set to 


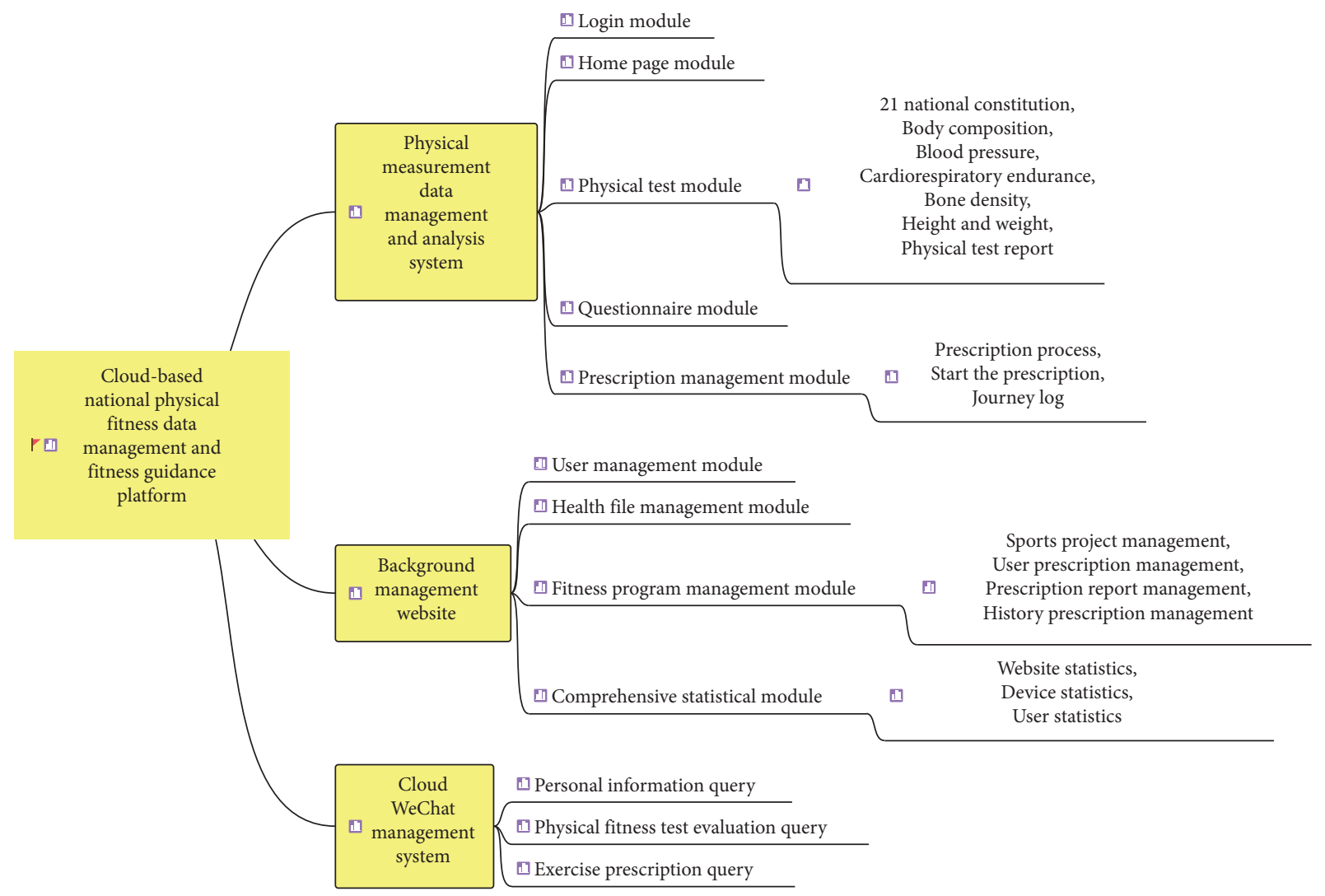

Figure 7: National fitness item recommendation system based on a neural network algorithm.

store the top k most similar fitness users of the target fitness user $U_{i}$. Therefore, $\left|S_{u_{i}}\right|=k$, a recommended list of target fitness user $u_{i}$. The detailed steps are as follows:

(1) The algorithm constructs a set $C P_{u_{i}}$, which contains all the POIs visited by fitness users in $S_{u_{i}}$.

(2) The algorithm sets the distance threshold $\lambda$ to determine the recommended area. The recommended area is a circle with the current position of the target fitness user $u_{i}$ as the center and $\lambda$ as the radius.

(3) For each sign-in point $p_{m}\left(p_{m} \in C P_{u_{i}}\right)$, we set the formula:

$$
\Phi\left(l, p_{m}\right)= \begin{cases}\lambda^{-1}, & d_{\left(l, p_{m}\right)} \leq \lambda, \\ d_{\left(l, p_{m}\right)}^{-1}, & d_{\left(l, p_{m}\right)}>\lambda .\end{cases}
$$

Specifically, $l$ is the current position of the fitness user $u_{i} \cdot d_{\left(l, p_{m}\right)}$ represents the Euclidean distance between $l$ and $p_{m}$.

(4) The algorithm uses formula (15) to calculate the pseudoscoring:

$$
\operatorname{score}\left(u_{i}, p_{m}\right)=\sum_{k=1}^{S_{u_{i}}}\left(\operatorname{simuser}\left(u_{i}, u_{k}\right) f\left(u_{k}, p_{m}\right) \Phi\left(l, p_{m}\right)\right) \text {. }
$$

Among them, $f\left(u_{k}, p_{m}\right)$ represents the pseudoscore of the fitness user $u_{k}\left(u_{k} \in S_{u_{i}}\right)$ to the check-in point $p_{m}$, which is counted by

$$
f\left(u_{k}, p_{m}\right)=\frac{N\left(u_{k}, p_{m}\right)}{\left|L_{k}^{q}\right|} .
$$

Among them, $f\left(u_{k}, p_{m}\right)$ represents the number of visits by the fitness user $u_{k}$ to check-in point $p_{m}$, and $f\left(u_{k}, p_{m}\right)$ represents the total number of check-in points visited by the fitness user $u_{k}$ in a time interval $T_{q}$.

(5) After obtaining the pseudoscoring of each POI in $C P_{u}$, the algorithm arranges in descending order and recommends the first $k$ POIs.

\section{National Fitness Item Recommendation System Based on a Neural Network Algorithm}

Based on the above neural network recommendation algorithm, the nationwide fitness item recommendation system based on the neural network algorithm constructed in this article is shown in Figure 7.

The design of the overall service process of the platform is shown in Figure 8:

After the above model is constructed, the effect of the model is evaluated. First, the effect of neural network algorithm fitness data processing is evaluated, and the result is shown in Figure 9. 


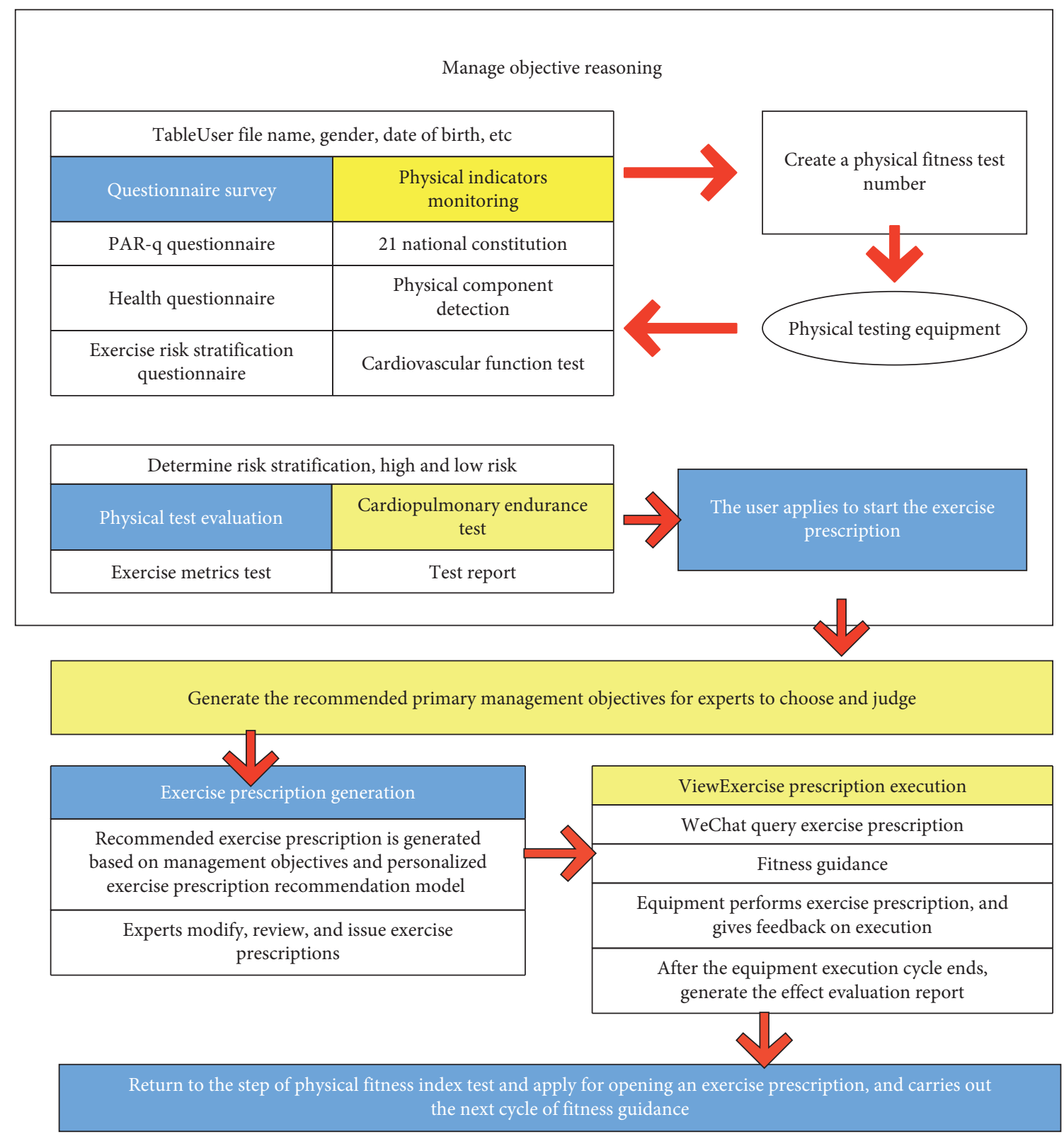

FIGURE 8: The overall service process of the platform.

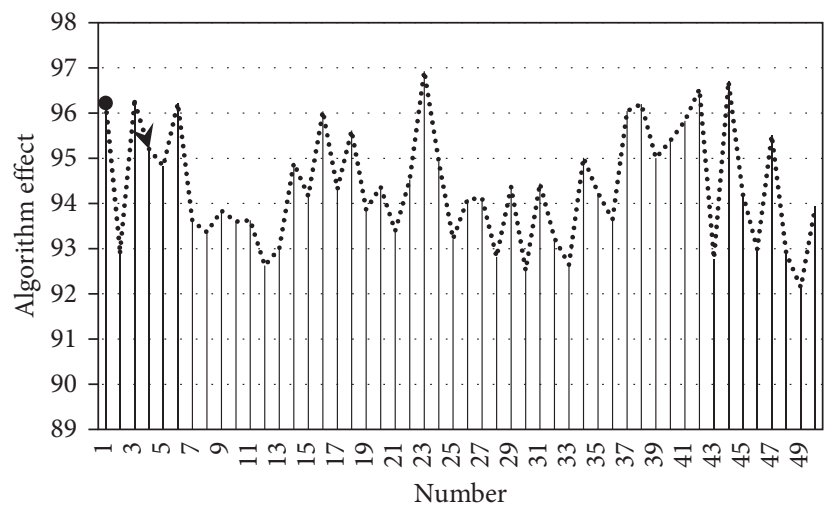

FIGURE 9: Neural network algorithm data processing. 


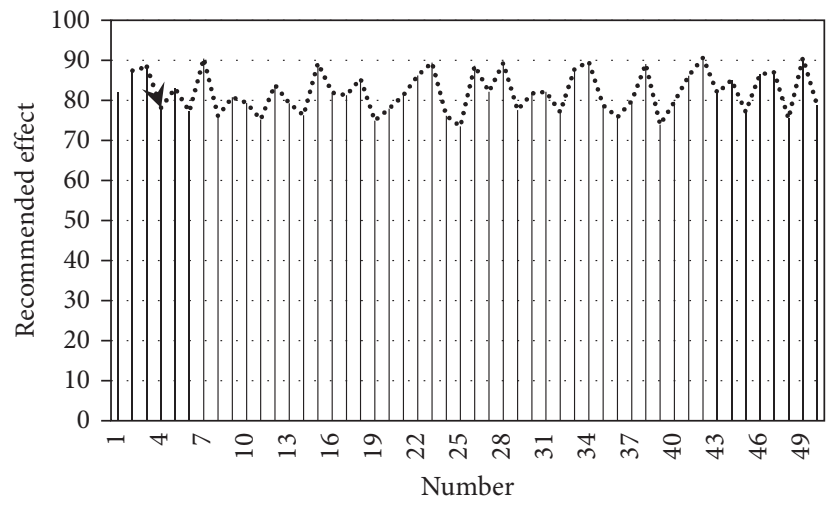

FIGURE 10: Recommendation effect of national fitness items based on the neural network algorithm.

It can be seen from the above research that the neural network algorithm proposed in this paper has a good data processing effect on the fitness data. On this basis, the effect of the national fitness item recommendation of the system in this paper is verified, and the result is shown in Figure 10.

From the above research, it can be known that the recommendation method of national fitness items based on the neural network algorithm has a certain effect.

\section{Conclusion}

National fitness activities have developed rapidly, and related achievements in the field of sports have entered a new level. At this stage, the problem that China faces in national fitness is the uneven development of public services. The main task at this stage is to use the construction of public service facilities to solve the problems and contradictions in China's fitness industry. Secondly, China's social public undertakings in the fields of education, culture, health, etc. have initially established competitive sports activities to win glory for the national Olympics. However, the public service system in the field of national fitness is relatively backward and to a certain extent cannot meet the national demand for physical fitness. According to the actual needs of the national fitness program, this paper combines the neural network algorithm to construct the national fitness program recommendation system to improve the recommendation efficiency of the national fitness program. The experimental research results verify that the method proposed in this article has a good performance in the recommendation efficiency of national fitness programs.

\section{Data Availability}

The labeled dataset used to support the findings of this study is available from the corresponding author upon request.

\section{Conflicts of Interest}

The authors declare that there are no conflicts of interest.

\section{Acknowledgments}

The research was supported by a project supported by Hunan Social Science Achievements Evaluation Committee in 2021 (No. XSP21YBZ061).

\section{References}

[1] A. H. Riley and C. Callahan, "Shoulder rehabilitation protocol and equipment fit recommendations for the wheelchair sport athlete with shoulder pain," Sports Medicine and Arthroscopy Review, vol. 27, no. 2, pp. 67-72, 2019.

[2] C. A. Emery and K. Pasanen, "Current trends in sport injury prevention," Best Practice \& Research Clinical Rheumatology, vol. 33, no. 1, pp. 3-15, 2019.

[3] V. M. Shulyatyev and M. A. Bulavina, "Sport interview: strategy, design and content," Theory and Practice of Physical Culture, vol. 7, no. 9, p. 18, 2019.

[4] L. J. Ferguson, K. T. Carlson, and D. Rogers, "Moving towards reconciliation through sport: sharing our process of exploring team saskatchewan experiences at the North American indigenous games," Journal of Exercise, Movement, and Sport (SCAPPS refereed abstracts repository), vol. 51, no. 1, p. 99, 2019.

[5] K. Kimasi, V. Shojaei, and M. R. Boroumand, "Investigation of safety conditions at gymnasia in different organizations," Journal of Humanities Insights, vol. 3, no. 2, pp. 70-74, 2019.

[6] K. Reinhart and B. Wichmann, "The TuS Fortschritt Magdeburg-Neustadt (soccer section) in the GDR - an example of amateur socialist sport," Soccer and Society, vol. 21, no. 4, pp. 408-420, 2020.

[7] C. Abanazir, "E-sport and the EU: the view from the English bridge union," The International Sports Law Journal, vol. 18, no. 3, pp. 102-113, 2019.

[8] A. Gerke, K. Babiak, G. Dickson, and M. Desbordes, "Developmental processes and motivations for linkages in crosssectoral sport clusters," Sport Management Review, vol. 21, no. 2, pp. 133-146, 2018.

[9] A. I. Pogrebnoy and I. O. Komlev, "Sport institutions reporting to Ministry of Sport of Russian Federation: intellectual property, invention activity, patenting and legal consulting service analysis," Theory and Practice of Physical Culture, vol. 23, no. 2, pp. 6-8, 2018.

[10] D. C. Ilies, R. Buhas, and M. Ilies, "Sport activities and leisure in Nature 2000 protected area-Red Valley, Romania," Journal 
of Environmental Protection and Ecology, vol. 19, no. 1, pp. 367-372, 2018.

[11] A. I. Kondrukh, "Practical shooting sport in Russian sport system: essential specifications and features," Theory and Practice of Physical Culture, vol. 21, no. 5, pp. 75-77, 2017.

[12] R. Giulianotti and D. Numerato, "Global sport and consumer culture: an introduction," Journal of Consumer Culture, vol. 18, no. 2, pp. 229-240, 2018.

[13] A. G. Gurinovich and G. V. Petrova, "Key priorities of physical education and sport sector budgeting laws and regulations in the Russian Federation," Theory and Practice of Physical Culture, vol. 6, no. 4, p. 34, 2019.

[14] M. Mountjoy, A. Costa, R. Budgett et al., "Health promotion through sport: international sport federations' priorities, actions and opportunities," British Journal of Sports Medicine, vol. 52, no. 1, pp. 54-60, 2018.

[15] J. J. Pulido, D. Sánchez-Oliva, P. A. Sánchez-Miguel, D. Amado, and T. García-Calvo, "Sport commitment in young soccer players: a self-determination perspective," International Journal of Sports Science \& Coaching, vol. 13, no. 2, pp. 243-252, 2018.

[16] J. Cristiani, J. C. Martins-Bressan, B. Longarela, L. Galatti, and R. Reverdito, "Clubs SOCIO-deportivos en UN municipio brasileño: ESPACIO, EQUIPOS Y CONTENIDOS [Sport clubs in Brazil: facilities, equipment and content in][Clubes socio-esportivos em município brasileiro: espaço, equipamentos e conteúdos]. E-balonmano. com," Revista de Ciencias del Deporte, vol. 13, no. 2, pp. 105-112, 2017.

[17] E. Happ, M. Schnitzer, and M. Peters, "Sport-specific factors affecting location decisions in business to business sport manufacturing companies: a qualitative study in the Alps," International Journal of Sport Management and Marketing, vol. 21, no. 1-2, pp. 21-48, 2021.

[18] M. Castro-Sánchez, F. Zurita-Ortega, and R. ChacónCuberos, "Motivation towards sport based on sociodemographic variables in university students from Granada," Journal of Sport and Health Research, vol. 11, no. 1, pp. 55-68, 2019.

[19] S. M. Hadlow, D. Panchuk, D. L. Mann, M. R. Portus, and B. Abernethy, "Modified perceptual training in sport: a new classification framework," Journal of Science and Medicine in Sport, vol. 21, no. 9, pp. 950-958, 2018.

[20] J. H. Du Plessis and M. Berteanu, "The importance of prosthetic devices in sport activities for Romanian amputees who compete in Paralympic competitions," Medicina Sportiva: Journal of Romanian Sports Medicine Society, vol. 16, no. 1, pp. 3197-3204, 2020. 